OPEN ACCESS

Edited by:

Jiajie Diao,

University of Cincinnati, USA

Reviewed by:

Min Liu,

Harvard Medical School, USA

Zhitao Hu,

University of Queensland, Australia

Zhiyong Shao,

Fudan University, China

*Correspondence:

Tao Xu

xutao@ibp.ac.cn

Lijun Kang

kanglijun@zju.edu.cn

${ }^{\dagger}$ These authors have contributed equally to this work.

Received: 28 February 2017 Accepted: 25 April 2017 Published: 11 May 2017

Citation:

Han M, Zou W, Chang H, Yu Y, Zhang $H$, Li S, Cheng H, Wei G, Chen $Y$, Reinke $V, X U T$ and Kang $L$ (2017) A Systematic RNAi Screen

Reveals a Novel Role of a Spindle Assembly Checkpoint Protein BuGZ in Synaptic Transmission in C. elegans.

Front. Mol. Neurosci. 10:141 doi: 10.3389/fnmol.2017.00141

\section{A Systematic RNAi Screen Reveals a Novel Role of a Spindle Assembly Checkpoint Protein BuGZ in Synaptic Transmission in C. elegans}

\author{
Mei Han ${ }^{1,2,3+}$, Wenjuan Zou ${ }^{1+}$, Hao Chang ${ }^{2,3+}$, Yong $\mathrm{Yu}^{2 \dagger}$, Haining Zhang ${ }^{2}$, Shitian $\mathrm{Li}^{1}$, \\ Hankui Cheng ${ }^{1}$, Guifeng $\mathrm{Wei}^{2}$, Yan Chen ${ }^{2}$, Valerie Reinke ${ }^{3}$, Tao $\mathrm{Xu}^{2 *}$ and Lijun Kang ${ }^{1 *}$ \\ ${ }^{1}$ Key Laboratory of Medical Neurobiology of the Ministry of Health of China, Department of Neurobiology, Institute of \\ Neuroscience, Zhejiang University School of Medicine, Hangzhou, China, ${ }^{2}$ National Laboratory of Biomacromolecules, CAS \\ Center for Excellence in Biomacromolecules, Institute of Biophysics, Chinese Academy of Sciences, Beijing, China, \\ ${ }^{3}$ Department of Genetics, Yale University School of Medicine, New Haven, CT, USA
}

Synaptic vesicles (SV) store various neurotransmitters that are released at the synapse. The molecular mechanisms of biogenesis, exocytosis, and endocytosis for SV, however, remain largely elusive. In this study, using Complex Object Parametric Analysis and Sorter (COPAS) to monitor the fluorescence of synapto-pHluorin $(\mathrm{SpH})$, we performed a whole-genome RNAi screen in C. elegans to identify novel genetic modulators in SV cycling. One hundred seventy six genes that up-regulating $\mathrm{SpH}$ fluorescence and 96 genes that down-regulating $\mathrm{SpH}$ fluorescence were identified after multi-round screen. Among these genes, B0035.1 (bugz-1) encodes ortholog of mammalian $\mathrm{C} 2 \mathrm{H} 2$ zinc-finger protein BuGZ/ZNF207, which is a spindle assembly checkpoint protein essential for mitosis in human cells. Combining electrophysiology, imaging and behavioral assays, we reveal that depletion of BuGZ-1 results in defects in locomotion. We further demonstrate that BuGZ-1 promotes SV recycling by regulating the expression levels of endocytosis-related genes such as rab11.1. Therefore, we have identified a bunch of potential genetic modulators in SV cycling, and revealed an unexpected role of BuGZ-1 in regulating synaptic transmission.

Keywords: RNAi screen, synaptic transmission, C. elegans, $\mathrm{C2H} 2$ zinc-finger protein, synaptic vesicles

\section{INTRODUCTION}

Synaptic vesicles (SV) store neurotransmitters, concentrate in the presynaptic nerve terminals, and undergo $\mathrm{Ca}^{2+}$-dependent exocytosis. These steps include biogenesis of SVs, transport to release sites, docking with plasma membrane, priming, and calcium-triggered fusion (Sudhof and Rizo, 2011; Rizo and $\mathrm{Xu}$, 2015). After exocytosis, SVs undergo endocytosis, recycle, and refilling with neurotransmitters for next round of exocytosis (Wu et al., 2014; Rizo and Xu, 2015; Xie et al., 2017). Three modes of exocytosis, including full-collapse fusion, kiss-and-run, and compound exocytosis, are coupled to classical endocytosis, kiss-and-run, and bulk endocytosis, respectively (Wu et al., 2014; Rizo and Xu, 2015; Xie et al., 2017).

The fusion of SVs to the plasma membrane is mechanically driven by the interaction among SNARE complex, a four-helix coiled-coil structure, which consists of a vesicle SNARE (v-SNARE) protein, synaptobrevin (on vesicle membrane), and two target membrane SNARE (t-SNARE) 
proteins, syntaxin-1, and SNAP-25 (on the plasma membrane) (Sudhof and Rizo, 2011; Südhof, 2013; Rizo and Xu, 2015). Fusion-competent conformations of SNARE proteins are maintained by chaperone complexes including $\operatorname{CSP} \alpha, \mathrm{Hsc} 70$, and SGT (Sudhof and Rizo, 2011). The synaptic SNARE and SM fusion-machine is controlled by synaptotagmin by $\mathrm{Ca}^{2+}$ via synaptotagmin and complexin, and is additionally regulated by a presynaptic active zone proteins that includes Munc13 and RIM as central components (Sudhof and Rizo, 2011). Classical endocytosis is clathrin-dependent (Wu et al., 2014). All three SNARE proteins that catalyze exocytosis-synaptobrevin, SNAP25, and syntaxin are also needed for endocytosis initiation (Sudhof and Rizo, 2011; Wu et al., 2014; Xie et al., 2017). Some molecules such as amphiphysin, endophilin, AP180, auxilin, and dynamin have been implicated to be involved in endocytosis ( $\mathrm{Wu}$ et al., 2014). Additionally, endocytosis is prolonged by depletion of clathrin, AP2, stonin 2, endophilin, and auxilin (Wu et al., 2014). Although the principle steps and some molecules have been identified, the exact mechanisms of biogenesis, exocytosis and endocytosis of SVs remain largely elusive.

Synaptobrevin is the key molecule on SVs, so synaptopHluorin $(\mathrm{SpH})$, a $\mathrm{pH}$-sensitive variant of GFP (pHluorin) fused to the luminal domain of synaptobrevin, is widely used to quantitatively measure the exocytosis and endocytosis of SVs (Miesenböck et al., 1998; Sankaranarayanan et al., 2000; Dittman and Kaplan, 2006; Afuwape and Kavalali, 2016). At rest, $\mathrm{SpH}$ fluorescence is quenched by the luminal acidic $\mathrm{pH}$ of the vesicle. After stimulation, vesicles fuse with the plasma membrane exposing the lumen to the neutral $\mathrm{pH}$ of the extracellular medium and causing an increase in $\mathrm{SpH}$ fluorescence. The fluorescence is then quenched once again after endocytosis and reacidification (Sankaranarayanan et al., 2000; Afuwape and Kavalali, 2016).

C. elegans is an excellent model system for studying SV cycling and performing systematic RNAi screen (Richmond and Broadie, 2002; Jadiya et al., 2016). With the availability of the C. elegans whole-genome RNAi feeding library, the expression of endogenous genes can be specifically knocked down by feeding bacteria expressing double-stranded RNA (dsRNA) of corresponding genes (Kamath et al., 2003). In order to get a clearer picture of the SV cycling, we aimed to identify novel genes required for synaptic vesicle cycling via C. elegans whole-genome RNAi screen, using $\mathrm{SpH}$ as the probe. Previous whole genome RNAi screens in C. elegans mostly detected and scored phenotypes by eyes, with limited quantifiable results, quality control, and systematic analysis. In this study, we detected the $\mathrm{SpH}$ fluorescence intensity of individual worms via Complex Object Parametric Analysis and Sorter (COPAS, Union Biometrica), which provides a method for high-throughput, reproducible quantitative analysis (Pulak, 2006; Dupuy et al., 2007). We screened two RNAi feeding libraries, including both Ahringer Library and Vidal Library (Kamath et al., 2003; Rual et al., 2004; Kim et al., 2005), together covering $94 \%$ of predicted C. elegans genes. We identified 176 genes up-regulating $\mathrm{SpH}$ fluorescence and 96 genes downregulating $\mathrm{SpH}$ fluorescence after multi-round screen. Among these candidate genetic modulators of SV cycling, the $\mathrm{C} 2 \mathrm{H} 2$ zinc-finger protein BUGZ-1, an ortholog of mammalian spindle assembly checkpoint protein BuGZ/ZNF207, is critically required for SV cycling, suggesting an unidentified role of spindle assembly checkpoint proteins in synaptic transmission.

\section{RESULTS}

\section{A Whole-Genome RNAi Screen Identifies Novel Genes Required for Synaptic Vesicle Cycling}

To identify novel genes related to synaptic vesicle (SV) cycling, we performed an automatic whole-genome RNAi screen in $C$. elegans. We used a pan-neuronal expressed $s n b-1$ promoter to drive $S N B-1::$ pHluorin $(\mathrm{SpH})$ expression in the nerve system for optical measurements of presynaptic activity. $\mathrm{SpH}$ is a $\mathrm{PH}$-sensitive variant of GFP fused to the luminal domain of synaptobrevin. Previous studies have confirmed that the fluorescence of $\mathrm{SpH}$ is quenched in the acidic environment of the SV lumen but increased dramatically when the SVs fused to the plasma membrane (Miesenböck et al., 1998; Sankaranarayanan et al., 2000; Dittman and Kaplan, 2006; Afuwape and Kavalali, 2016). The transgenic strain Is[snb-1::pHluorin] was crossed with the RNAi hypersensitive strain KP3948 eri-1(mg366); lin$15 b(n 744)$ (Sieburth et al., 2005) to generate worm for wholegenome RNAi screen. We performed screen with COPAS, a machine which is able to effectively detect the changes of fluorescence intensity in C. elegans by line scanning, and profile the fluorescent intensity for each worm (Figure S1, Dupuy et al., 2007; Han et al., 2013). For high-throughput screen using COPAS, worms were incubated in liquid culture in standard flat-bottomed 96-well plates according to previous reports with some modification (Lehner et al., 2006). We developed series of programs to batch process the raw data of COPAS. Relative florescent signals (RFS) were used to represent the $\mathrm{SpH}$ fluorescent signal, and robust Z-score were calculated to normalize $\mathrm{SpH}$ fluorescent intensity from different experimental 96-well plates (see Materials and Methods).

We chose unc-11/AP180, which has been reported to play an important role in recycling synaptobrevin from plasma membrane (Nonet et al., 1999; Dittman and Kaplan, 2006; Sudhof and Rizo, 2011), as an up-regulated control (up regulate $\mathrm{SpH}$ fluorescent intensity). Knocking down unc-11 AP180 6-fold increased the fluorescent intensity of $\mathrm{SpH}$ in the probe strain (Figure S1). We used the RNAi bacteria expressing dsRNA of $g f p$, whose sequence is similar to pHluorin, as a down-regulated control (down regulate $\mathrm{SpH}$ fluorescent intensity). Knocking down pHluorin by feeding worms with $g f p$ RNAi bacteria decreased the fluorescent intensity of $\mathrm{SpH}$ to $<0.5$-fold in worms. The fluorescent intensities of worms cultured in 96-well plates in liquid which detected by COPAS were consistent with the fluorescent intensities of worms cultured on RNAi plates which detected by the confocal microscope. These results indicated that positive genes can be knocked down by feeding worms with corresponding RNAi bacteria in liquid culture, and the changes of fluorescent intensity can be detected efficiently by the COPAS.

In order to monitor the quality of RNAi treatment for each 96well plate, we added up-regulated control of unc-11 RNAi clones, 
down-regulated control of $g f p$ RNAi clones, and empty vector control of L4440 clones into the empty wells of each 96-well plate. We rearranged a few clones in 96-well plates to insure each experimental plate contains two empty vector controls, one upregulated control and one down-regulated control. Synchronized L1 worms were fed with each RNAi clone in each well of 96well plates, and $\mathrm{SpH}$ fluorescence was detected in their progeny via the COPAS. For those RNAi clones that caused embryonic lethal or sterile, $\mathrm{SpH}$ fluorescence was detected in young adults of the same generation (Figure 1A). All bacteria RNAi clones were duplicated in the whole-genome screen. The plates with low repeatability (fold change of the florescent signals between the two repeats larger than 1.5) were redone. We examined the repeatability of two repeats for each clone, the overall correlation of the paired repeats is 0.81 , which means that most paired repeats have very similar values (Figure 1B). Based on the genomic distribution of fluorescent intensity change after RNAi treatment, genes fell in the two tails (5\%) were chosen as candidates. The candidates were rearranged into new 96well plates with controls for further validation. In the secondary validation, worms were retested under the same condition. Each RNAi clones had four repeats. Genes that caused stable and consistent $\mathrm{SpH}$ signal changes were chosen as positive hits.

The fluorescent intensity changes of $\mathrm{SpH}$ could also due to unspecific reasons, especially the changing of protein expression level, so it is necessary to exclude unrelated genes for SV cycle. We checked the expression level of GFP in neurons, using the RNAi hypersensitive worm strain nre-1(hd20); lin15b(hd126);rhIs13[unc-119p::GFP + dpy-20(+)] (Schmitz et al., 2007). Genes that lead to significant fluorescent intensity changes $(p<0.05)$ were excluded. Finally, 176 genes with up-regulated fluorescent intensity of $\mathrm{SpH}$ and 96 genes with down-regulated fluorescent intensity of $\mathrm{SpH}$ were identified after multi-round screen (Table S2). Seventy-six percent of these genes are evolutionarily conserved. The functional classes of candidate genes indicated that diverse groups of genes were taken part in SV cycling (Figure 1C and Table S3). Although some genes were already identified to be required for SV cycling, there still a big portion of genes with less known, which maybe new important regulators for SV cycling.

\section{Mutants of Candidate Genes Display Acetylcholine Release Defects}

In C. elegans, steady-state acetylcholine (ACh) secretion can be indirectly detected by measuring their resistance to the acetylcholine esterase inhibitor aldicarb (Mahoney et al., 2006b). Accumulation of ACh at synapsis caused by aldicarb leads to acute paralysis of worms and finally death. Blocking of SV cycle could lead to less release of ACh, which can be detected by aldicarb resistance analysis (Lackner et al.,

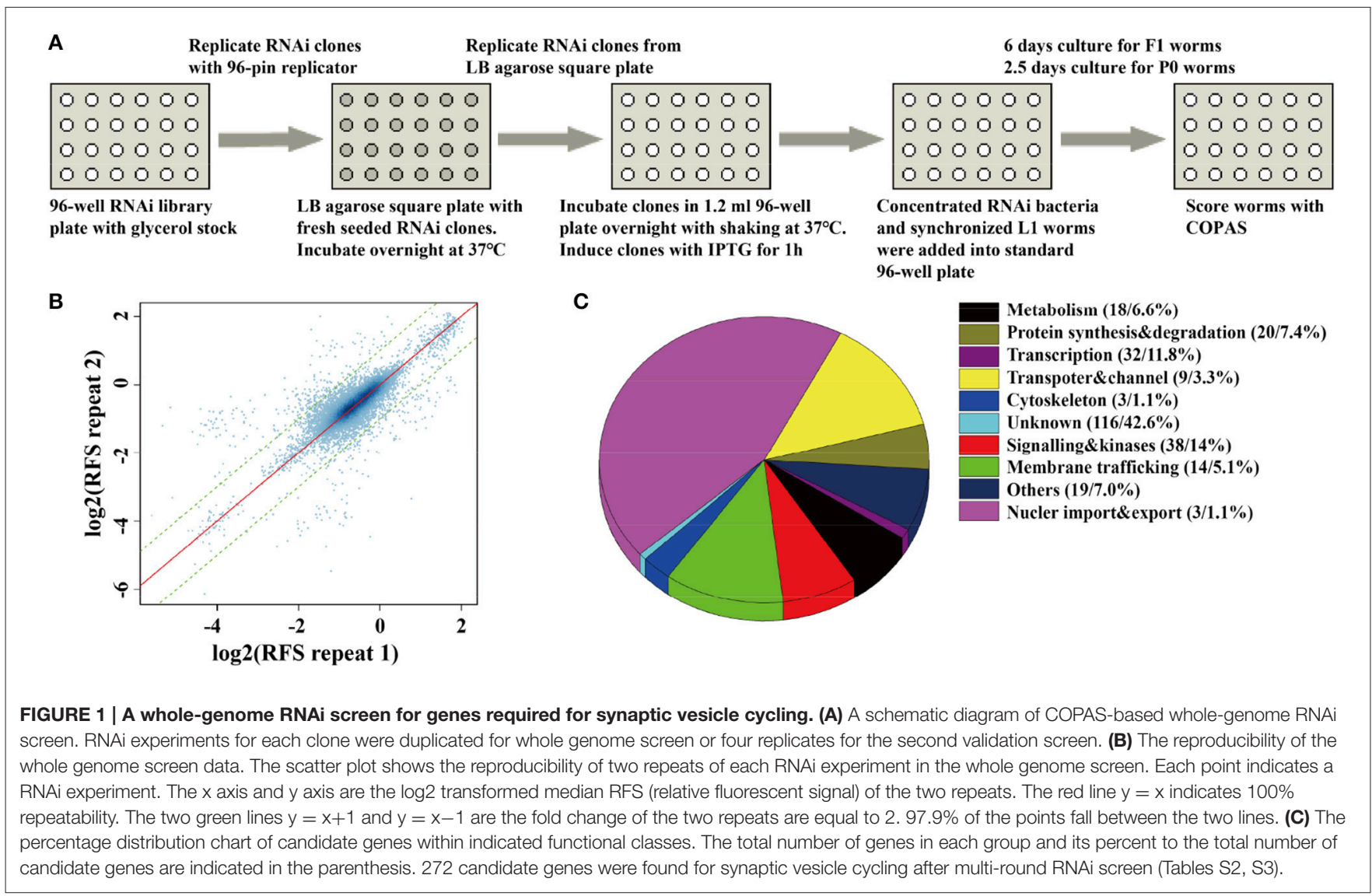


1999; Mahoney et al., 2006b). To validate the participation of candidates in neurotransmitter release, we examined the resistance to aldicarb in 14 mutations which lead to dramatic $\mathrm{SpH}$ fluorescent intensity changes when knocking down by RNAi treatment. We used unc-32(e189) as a positive control in aldicarb resistance assay (Wiese et al., 2012). unc-32 encodes an ortholog of subunit of the membrane-bound domain of vacuolar proton-translocating ATPase. unc-32(e189) is a specific mutant that is important for locomotion and SV morphology in motoneurons (Wiese et al., 2012). Nine mutants showed significant aldicarb-resistance phenotypes, including B0035.1(tm578), Y71G12B.11(ok1648), Y25C1A.7(tm2889), F41D9.3(ok695), F45E4.3(ok2285), Y76A2A.2(gk107), T23F11.1(tm3480), F59E12.11(tm3828), C18B12.2(tm1690)

(Figure 2A). The other five mutants showed no resistance (data not shown). All these candidate genes we identified are evolutionarily conserved, of which orthologs can be found in human, mouse, and fly, indicating their critical roles in diverse species.

In order to identify whether candidate genes function in presynaptic or postsynaptic terminal, we examined their resistance to the drug levamisole. Levamisole is a cholinergic receptor agonist that directly activates postsynaptic ACh receptors (Richmond and Jorgensen, 1999; Culetto et al., 2004). Wild type worms and mutants defected in presynaptic terminal display similar paralyzed phenotypes. All these 9 mutants exhibited similar paralysis rates with wild type (Figure S2), indicating a presynaptic function of candidate genes. Besides, $\mathrm{SpH}$ fluorescent intensities were significantly changed when knocking down these genes with RNAi treatment in the primary and the secondary screen, while GFP signals unaltered in nerve system of VH624 worms after RNAi treatment. These results also indicated that these genes probably function in presynaptic nerve terminal.

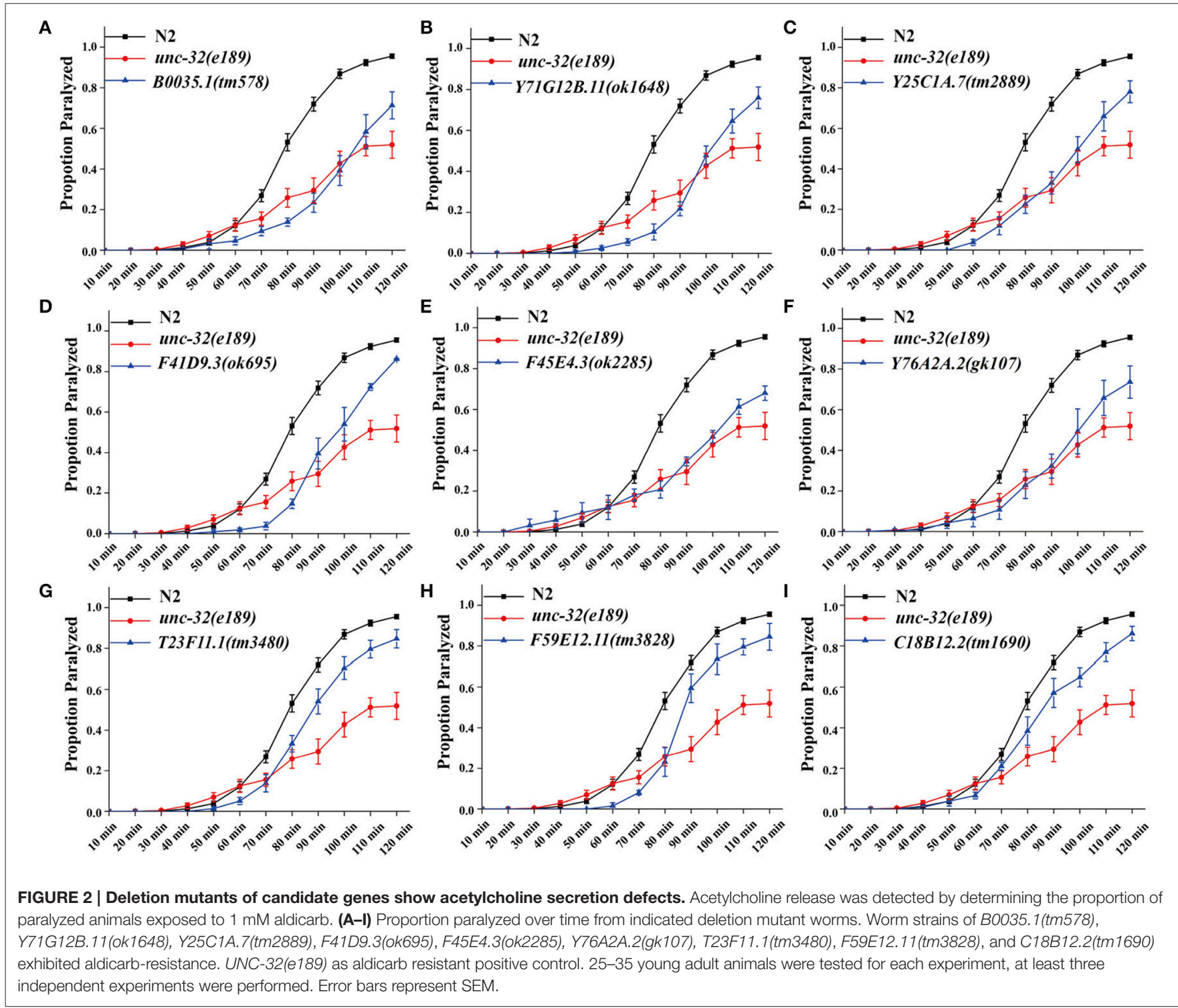




\section{BuGZ-1 Is a C2H2 Zinc-Finger Protein Expressed in the Nuclei of Neurons and Muscles}

Among these 9 candidate genes, B0035.1 (bugz-1) encodes an ortholog of mammalian $\mathrm{C} 2 \mathrm{H} 2$ zinc-finger protein BuGZ/ZNF207. Very interestingly, recent studies have been implicated that mammalian BuGZ binds to and stabilizes spindle check point protein Bub3 during interphase and facilitates mitosis (Jiang et al., 2014; Toledo et al., 2014). Depletion of BuGZ in cancer cells causes chromosome misalignment and mitotic arrest followed by massive cell death (Jiang et al., 2014;
Toledo et al., 2014). Our observation implicates that BUGZ may also have an unidentified role in synaptic transmission, thus we focused our following study on BUGZ-1. The C. elegans gene bugz-1 encodes two isoforms of BuGZ-1, BuGZ-1S (B0035.1a) and BuGZ-1L (B0035.1b), both containing a nuclear localization sequence (NLS) and two $\mathrm{C} 2 \mathrm{H} 2$ zinc finger domains at the $\mathrm{N}$-terminal of the protein (Figures 3A,B). BUGZ-1 is highly conserved from worm to human and exhibits $68 \%$ identity between amino acids 1 and 107 of mouse and human homologs (Figure 3C). We generated a bugz-1p::gfp construct by driven GFP under the promoter of the bugz-1 gene. GFP signals were observed throughout nervous system and muscles, including

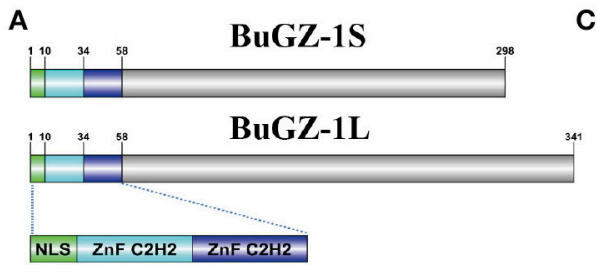

B

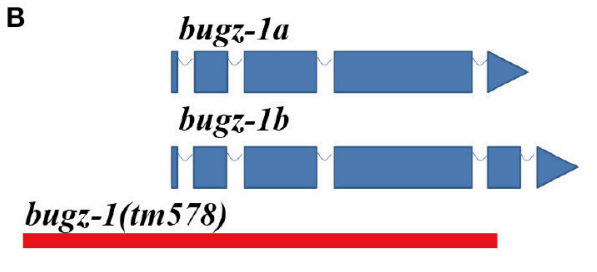

D
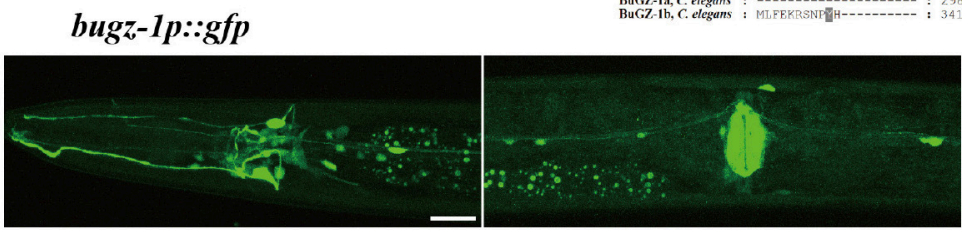

E

bugz-1::gfp; rab-3p::mCherry
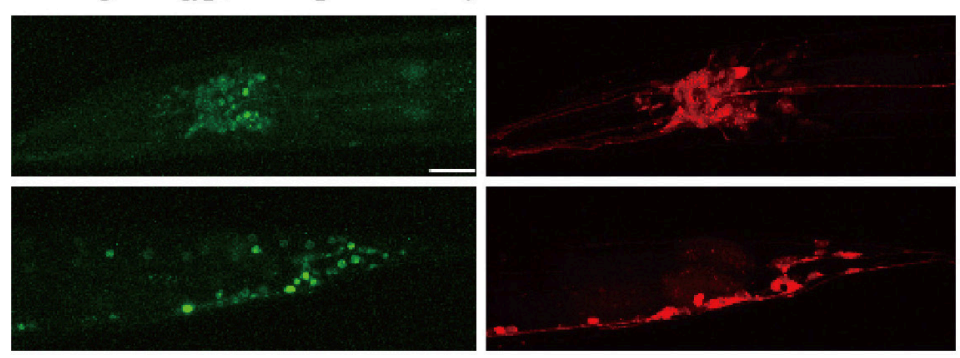

C
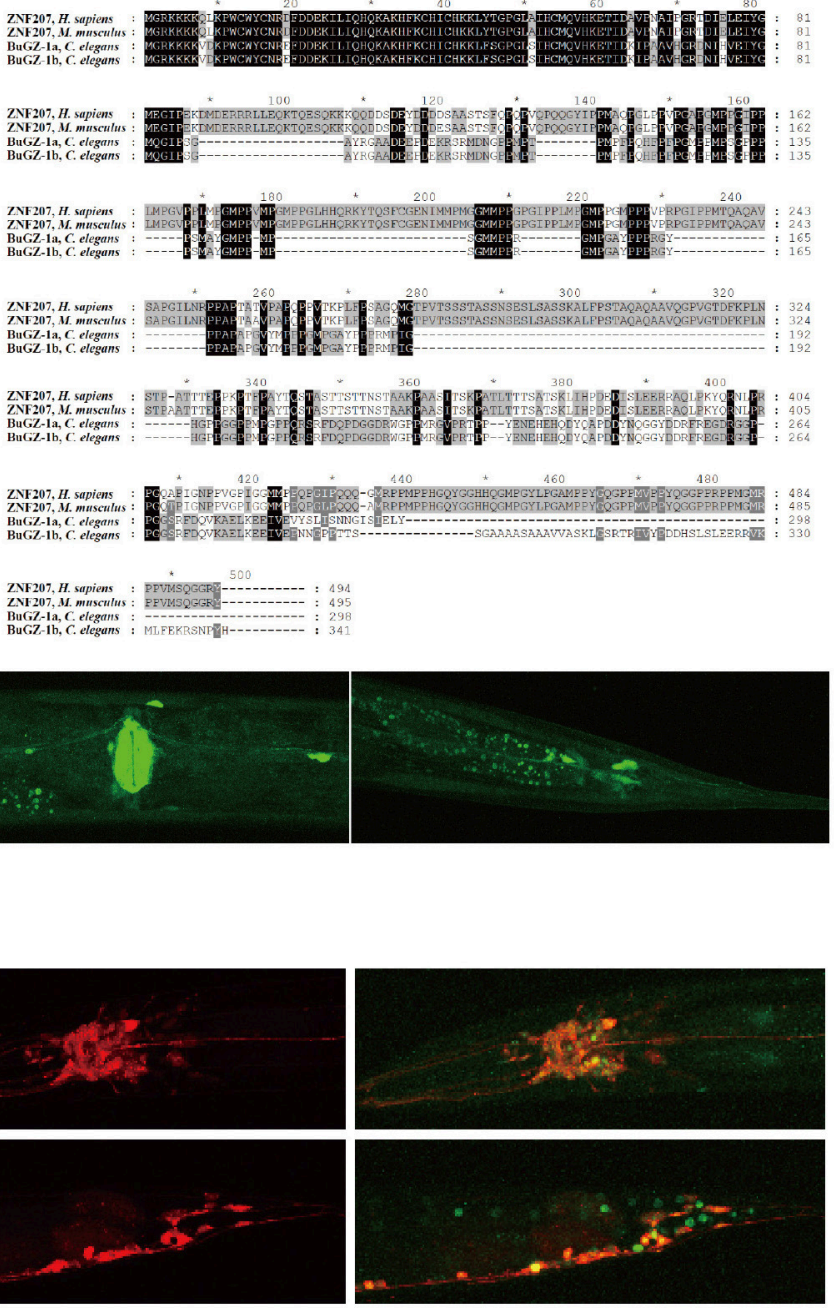

FIGURE 3 | BuGZ-1 is an evolutionary conserved protein with a predicted function as transcription factor and is mainly expressed in the nervous system. (A) Schematic diagram of protein domain structure of two isoforms of BuGZ-1. Both short isoform BuGZ-1S and long isoform BuGZ-1L have a nuclear localization signal (NLS) motif and two zinc finger $\mathrm{C} 2 \mathrm{H} 2$ (ZnF C2H2) domains. (B) Schematic diagram of gene structure for bugz-1a and bugz-1b. Blue boxes indicate exons; blue lines indicate introns. 8X backcross of bugz-1(tm578) to wild type was performed before doing experiments. (C) Multiple sequence alignment of the highly conserved N-terminal of BuGZ-1 from different species, including humans (H. sapiens), mouse (M. musculus), and worm (C. elegans). Perfect sequence conservation is indicated in black. (D) Transcriptional expression pattern of bugz-1p::gfp. bugz-1 is expressed in the nervous system, intestine and muscles. Images were taken from head, valve, and tail in ventral view, head to the left. (E) Translational expression pattern of bugz-1::gfp. Neurons are marked with rab-3p::mCherry. Images were taken from head and tail in lateral view, head to the left. BuGZ-1 is mainly localized to the nuclei of neurons. Scale bars indicate $20 \mu \mathrm{m}$. 
the nerve ring and ventral nerve cord (Figure 3D). In order to confirm the nervous system expression of BuGZ-1, we co-injected the constructs of bugz-1p::gfp and rab-3p::mCherry. BUGZ-1 is co-localized well with RAB-3 in most neurons in the nerve ring and the tail. Notably, BUGZ-1 is localized to the nuclei of these cells, consistent with the subcellular location of mammalian BuGZ (Figure 3E, Jiang et al., 2014; Toledo et al., 2014).

\section{Depletion of BuGZ-1 Results in Locomotion Defects}

To further investigate the function of BuGZ-1 in synaptic vesicle cycling, we used a mutant strain bugz-1(tm578) generated by the National Bioresource Project (Tokyo, Japan). Five out of six exons are deleted in bugz-1(tm578) mutant worms. The deletion starts from the promoter region of bugz-1, which makes bugz-1(tm578) a null allele (Figure 3B). While wild type animals exhibited a smooth and continuous sinusoidal movement, bugz-1(tm578) animals displayed more hesitating and uncoordinating movement with decreased trajectories and locomotion speed (Figures 4A,B). Moreover, mutants prefer to move backward (ratio of total time $=25.3 \pm 10.4 \%$ ) or stay immobile $(3.85 \pm 4.01 \%)$ when compared with wild type worms $(5.8 \pm 7.3 \%$ and $0.76 \pm 1.66 \%$, respectively) (Figures 4C,D).

\section{BuGZ-1 Regulates Neurotransmitter Release at NMJs}

C. elegans locomotion is controlled by nervous system that innervate specific muscles. Defects of either neuronal or muscle activities lead to locomotion abnormal (Richmond and Jorgensen, 1999; Dittman and Kaplan, 2006). We observed that bugz-1 mutants showed a dramatic resistance to aldicarb, an inhibitor for acetylcholine esterase (Dittman and Kaplan, 2006; Mahoney et al., 2006b, Figure 4E). The aldicarb-resistance phenotype and locomotion defects in bugz-1 mutants can be rescued by transgenic expression of BUGZ-1 cDNA driven by its own promoter or by the pan-neuronal promoter $r a b-3$ (Figures 4B-E, Mahoney et al., 2006a), suggesting that BuGZ-1 predominately functions in nervous system to regulate synaptic transmission.

We then directly measured neurotransmitters release by recording the endogenous excitatory postsynaptic currents (EPSC) and evoked EPSCs at NMJs in zxIs6 animals, in which a light-gated cation channel, channelrhodopsin-2 (ChR2), is expressed specifically in cholinergic motor neurons (Liewald et al., 2008; Yang et al., 2015). The evoked EPSCs were recorded in voltage-clamped muscles with $100 \mathrm{~ms}$ blue light stimulation. Endogenous EPSC of bugz-1 mutants decreased $50 \%$ in the frequency whereas the amplitudes of release events didn't altered, indicating a shrink in spontaneous release events (Figures 5A-C). The evoked response in bugz-1 mutants also displayed a 55\% decrease, suggesting a significant defect in regulated release (Figures 5D,E). The defects in both endogenous and evoked EPSCs were rescued by neuronal-expression of
BuGZ-1 (Figures 5A-E). Furthermore, $1 \mathrm{mM}$ acetylcholineinduced currents in body muscles didn't show any differences between wild type and bugz-1 mutant animals (Figure 5F). These results suggest that the decrease of EPSCs at NMJs is due to decreased neurotransmitter release from motor neurons rather than altered response to neurotransmitters of body muscles.

\section{BuGZ-1 Promotes Synaptic Vesicle Endocytosis}

The $\mathrm{SpH}$ fluorescence was largely increased when BuGZ1 was knocked down by RNAi treatment (1.43-fold change compared with L4440 empty vector). Significant increases of the $\mathrm{SpH}$ fluorescence were also observed in the nerve ring, the ventral nerve cord, as well as the axonal $\mathrm{SpH}$ puncta and inter-puncta of the dorsal nerve cord in bugz-1(tm578) mutations (Figure 6), suggesting an increase in SV exocytosis or a decrease in endocytosis in bugz-1(tm578) background. Given the neurotransmitter release was decreased at NMJs in bugz1 mutant animals (Figure 5), we propose BuGZ-1 regulates synaptic transmission by promoting SV recycling, particularly endocytosis.

\section{BuGZ-1 Regulates the Expression Levels of Endocytic Genes}

The nuclei expression pattern of BuGZ-1 suggested that this protein may regulate neurotransmitter release by altering the expression level of some crucial genes related to synaptic vesicle recycling. Based on this speculation, we performed a high-throughput sequencing of $C$. elegans cDNA generated by isolating total RNA (RNA-seq) from wild type and bugz-1(tm578) worms (Table S4). We examined expression levels of coding sequence on a genome-wide and quantified the differences of expression levels for each gene between wild type and mutants. The expression levels of known genes essential for clathrinmediated endocytosis including unc-11/AP180, chc-1/clathrin, and snb-1/synaptobrevin (Sudhof, 2004; Wu et al., 2014) were significantly reduced in bugz-1(tm578) worms (Figure 7A). Rab11.1, a small GTPases Rab required for endocytic recycling in many eukaryotic species (Sato et al., 2008), also showed a reduced expression level in bugz-1(tm578) worms (Figure 7A). We asked whether BuGZ-1 and endocytic genes function sequentially in the recycling pathway or in parallel pathways. If they function in the same pathway, simultaneous knockout or knockdown would be expected to give a phenotype similar to single mutants of either gene. However, in bugz-1(tm578);unc-11(e47) double mutant worms, more significant increases of the $\mathrm{SpH}$ fluorescence were observed both in the nerve ring and the ventral nerve cord compared to either bugz-1(tm578) or unc-11(e47) single mutant worms. These strongly enhanced phenotypes in double mutants suggest that BuGZ-1 and UNC-11 mainly function in parallel pathways, though loss of BuGZ-1 may result in down-regulation of UNC-11 (Figures 7B,C). Notably, whereas rab-11.1(RNAi) shows similar phenotypes with increased $\mathrm{SpH}$ fluorescence with bugz-1(RNAi) worms, no synthetic effect was observed in bugz1(RNAi) combined with rab-11.1(RNAi) depletion, suggesting 
A

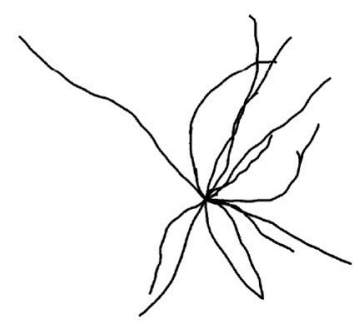

N2

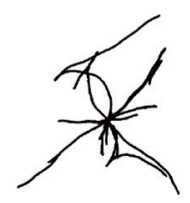

bugz-1(tm578)

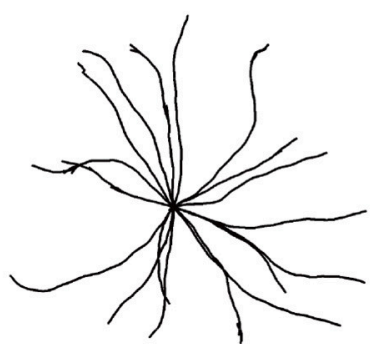

BuGZ-1 rescue
B

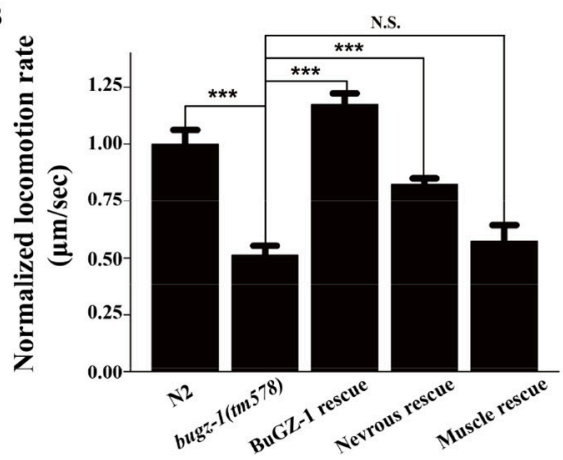

D

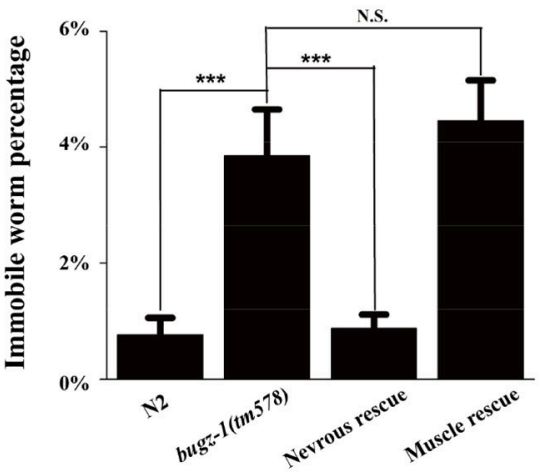

C

Muscle rescue

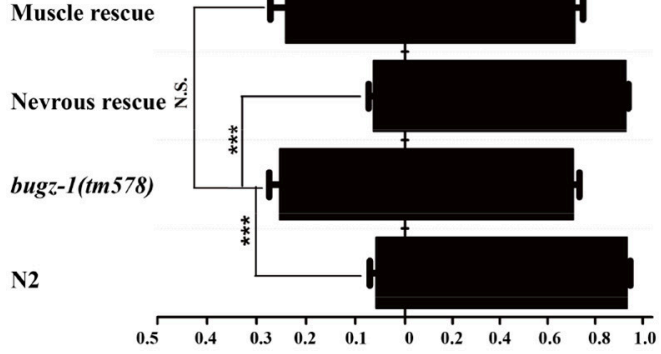

E

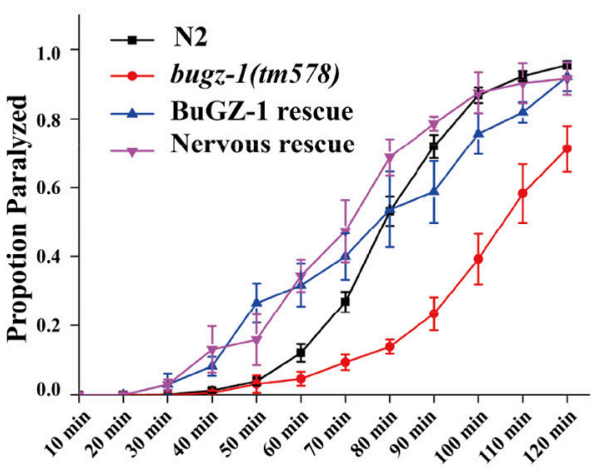

FIGURE 4 | BuGZ-1 regulates acetylcholine secretion and worm locomotion. (A,B) bugz-1(tm578) animals displayed more hesitating and uncoordinating movement with decreased trajectories (A) and locomotion speed (B). The locomotion rate is the average velocity of each worm in 40 seconds. (C,D) bugz-1(tm578) mutant worms prefer to move backward or stay immobile. (C) Statistic comparison of average proportion of time spent on moving forward or backward for indicated worms. (D) Percentage of worms stay immobile in indicated genotypes. All the locomotion defects of bugz-1(tm578) can be rescued by endogenous expressed BuGZ-1 (bugz-1::gfp) and nervous expressed BuGz-1 (rab-3p:::bugz-1::mCherry) but not muscle expressed BuGz-1 (myo-3p:::bugz-1::mCherry) in mutation background. The number of worms analyzed for locomotion is $n=15$ for each genotype. (E) Proportion paralyzed over time from indicated worm strains exposed to $1 \mathrm{mM}$ aldicarb. Aldicard resistance defect of bugz-1(tm578) was rescued by endogenous expressed BuGZ-1 and nervous expressed BuGZ-1 but not muscle expressed BuGZ-1 in mutation background. 25-35 young adult animals were tested for aldicarb resistant experiment, at least three independent experiments were performed. Values that significantly differ from controls are indicated ${ }^{\star \star \star} p<0.001$ by two-tails Student's $t$-test). N.S. indicates no significant difference from worm genotypes compared. Error bars represent SEM.

that BuGZ-1 and RAB-11.1 may function sequentially in the recycling pathway (Figures 7 D-F). Considering BuGZ-1 is a predicted transcription factor and has nuclei localization, we performed chromatin immunoprecipitation followed by deep sequencing (ChIP-seq) of single copy transgenic worm BuGZ$1:: g f p$ at young adult stage to determine binding sites of BuGZ-1 in the genome. We found that BuGZ-1 directly binds to rab-11.1 (Figure S3 and Table S5), indicates that BuGZ-1 may function in endocytosis by regulating RAB-11.1, which consistent with our conclusion that BuGZ-1 and RAB-11.1 may function sequentially in SV recycling pathway.

\section{DISCUSSION}

In this study, we performed a high-throughput and quantitative whole-genome RNAi screen in C. elegans using COPAS. We identified 176 genes with up-regulated $\mathrm{SpH}$ fluorescence and 
A

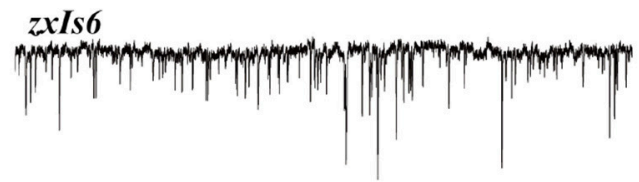

zxIs6; bugz-1(tm578)

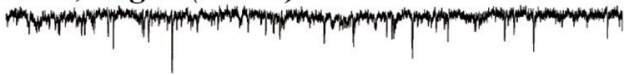

zxIs6; bugz-1(tm578); rab-3p::bugz-1::mCh

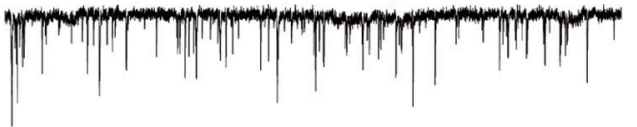

D

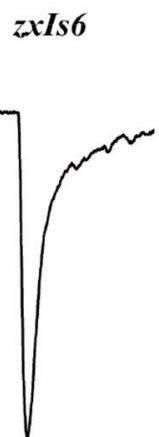

B

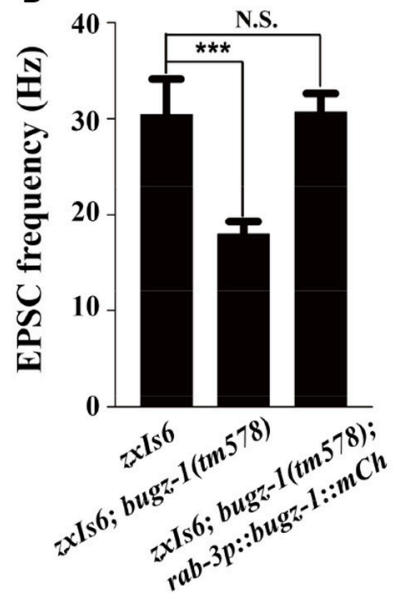

E

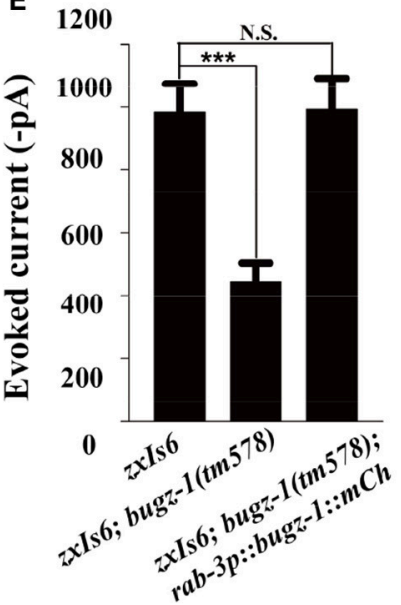

C

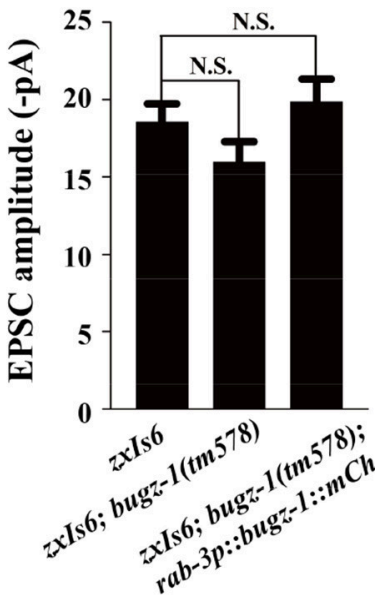

$\mathbf{F}$

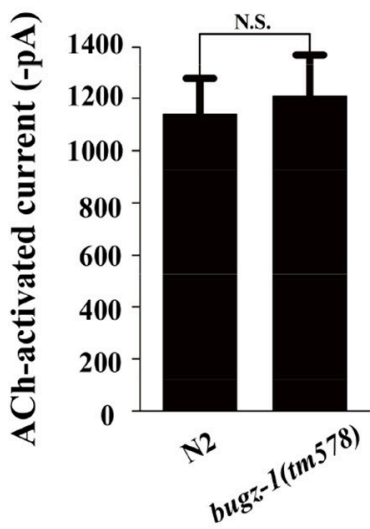

FIGURE 5 | BuGZ-1 regulates neurotransmitter release at NMJs. (A-C) Recording of endogenous excitatory postsynaptic currents (EPSC) from adult body wall muscles of the indicated genotypes. zxIs6 [unc-17p::chop-2(H134R)::yfp, lin-15(+)]. Representative traces (A) and summary data for endogenous EPSC frequency (B) and EPSC amplitude (C) are shown. $n \geq 15$. (D,E) Recording of photo-evoked EPSCs from adult body wall muscles of the indicated genotypes. ChR2-expressing cholinergic motor neurons were photo-activated by $100 \mathrm{~ms}$ blue light. Representative traces (D) and summary data (E) are shown. $n \geq 10$. (F) Acetylcholine-induced currents in body muscles of mutant were indistinguishable from wild type. $n \geq 10$. For each genotype, independent experiments were carried out in different animals. Values that significantly differ from controls are indicated $\left(^{\star \star \star} p<0.001\right.$ by two-tails Student's $t$-test). N.S. indicates no significant difference from control. Error bars represent SEM.

96 genes with down-regulated $\mathrm{SpH}$ fluorescence, which may be essential modulators for SV cycling. The functional classes of candidate genes indicated that diverse groups of genes, such as genes related to transcription, signaling, and kinase, transporter and channels, and membrane trafficking, are involved in SV cycling, supporting the vision that SV cycling is a complex and dynamic process involving a variety of mechanisms carried out by diverse groups of molecules.

Mammalian BuGZ/ZNF207 is a spindle assembly checkpoint protein associating with spindle microtubules and regulates chromosome alignment (Jiang et al., 2014; Toledo et al., 2014). Inhibition of BuGZ results in loss of both Bub3 and its binding partner Bub1 from kinetochores, and lethal chromosome congression defects in cancer cells (Jiang et al., 2014; Toledo et al., 2014). Nevertheless, a role of spindle assembly checkpoint proteins such as BuGZ in regulation of SV cycling have not been reported. By combining molecular genetics, optogenetics, electrophysiological recording and behavioral analysis, here we demonstrate that the C. elegans homolog of BuGZ is also a key modulator of synaptic vesicle recycling. Whether, BuGZ and other mitosis-associated proteins in other species have a role in synaptic transmission has yet to be identified. We envision that the functional characterization of C. elegans BuGZ may provide very useful insights into the functions of BuGZ and other mitosis-associated proteins in general.

The genetic interactions between bugz-1 and endocytic genes such as unc-11 and rab-11.1 strongly suggest that bugz-1 functions through, or in parallel to, clathrin-mediated endocytosis and vesicle recycling pathway during synaptic transmission. BuGZs are $\mathrm{C} 2 \mathrm{H} 2$ zinc finger proteins, which represent the second largest gene family in humans after the odorant receptor family (Tadepally et al., 2008). Most of the 

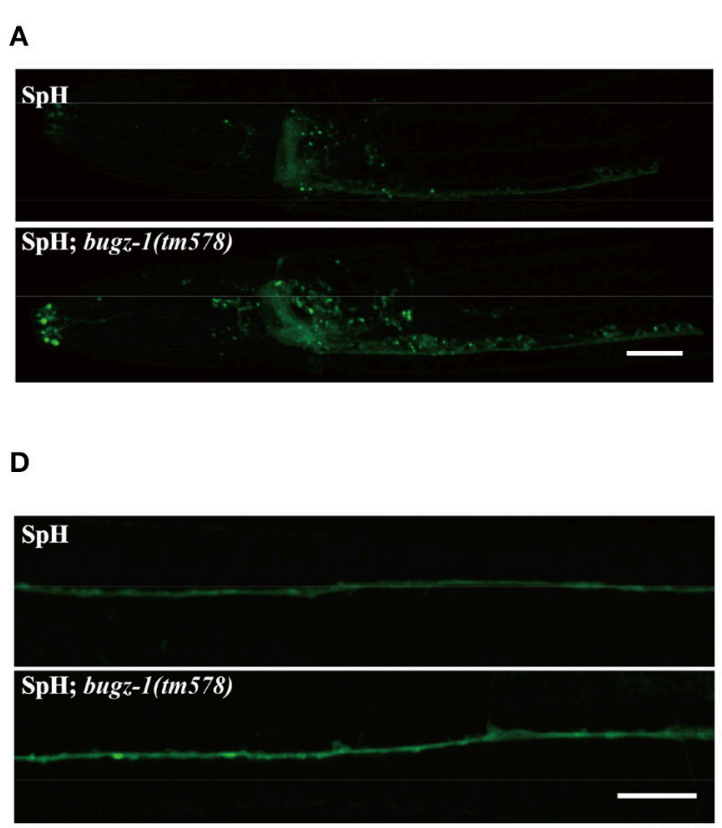
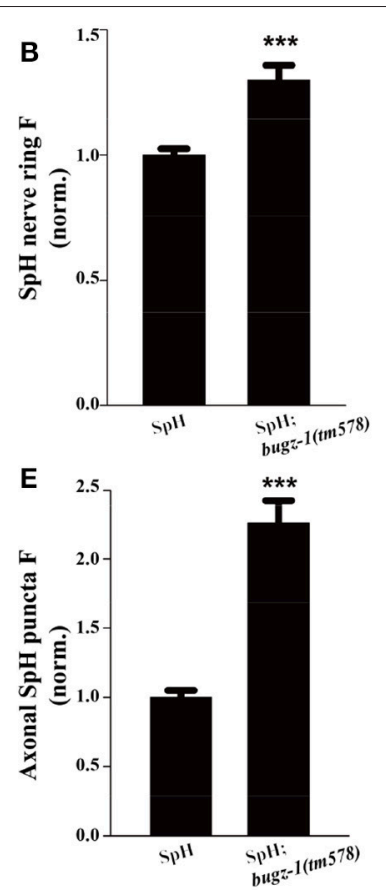
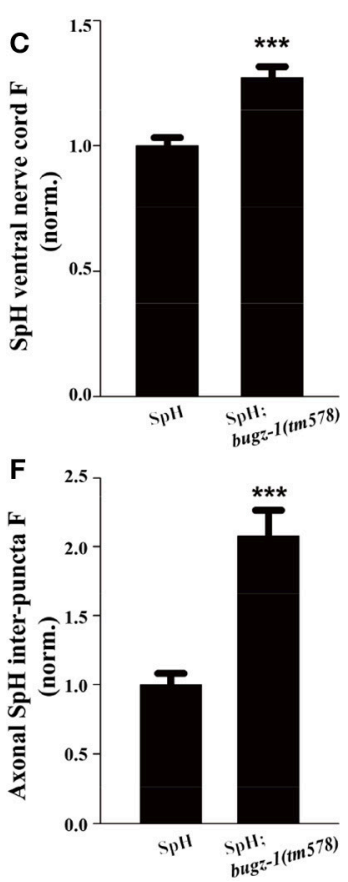

FIGURE 6 | bugz-1(tm578) show synaptic vesicle endocytosis defect. (A) Representative images of SNB-1::pHluorin (SpH) in the nerve ring and ventral nerve cord in wild type and bugz-1(tm578) mutant animals. (B,C) Quantification of fluorescence in the nerve ring (B) and ventral nerve cord (C) of bugz-1(tm578) mutant normalized to the wild-type control. $n \geq 14$. (D) Representative images of SpH in the dorsal nerve cord in wild type and bugz-1(tm578) mutant animals. (E,F) Quantification of axonal puncta fluorescence (E) and inter-puncta fluorescence (F) of dorsal nerve cord in mutant normalized to the wild-type control. $n \geq 7$. Values that significantly differ from controls are indicated ${ }^{\star \star \star} p<0.001$ by two-tails Student's $t$-test). Error bars represent SEM. Scale bars indicate $10 \mu \mathrm{m}$ in $\mathbf{( A )}$ and $20 \mu \mathrm{m}$ in (D).

characterized $\mathrm{C} 2 \mathrm{H} 2$ zinc finger genes code for transcription factors which bind DNA through their zinc finger region; others may bind RNA and protein motifs (Wang et al., 2006; Tadepally et al., 2008). However, their exact function is as yet unknown (Tadepally et al., 2008). Given that BuGZ-1 localizes to nuclear and down-regulates the expression level of some key endocytic proteins, it is likely that BuGZ-1 functions as a transcription factor or a posttranscriptional modulator for proteins essential for endocytosis. This transcriptional or posttranscriptional modulation may provide a fine tune mechanism for synaptic transmission.

Taken together, our study provides an example that performing a high-throughput and quantitative whole-genome RNAi screen in C. elegans, and identified diverse groups of molecules may be involved in SV cycling. These molecules may have a broader impact on protein and vesicle trafficking. Furthermore, we have directly correlated the role of a $\mathrm{C} 2 \mathrm{H} 2$ zinc finger spindle assembly checkpoint protein with synaptic transmission in an in vivo setting.

\section{MATERIALS AND METHODS}

\section{C. elegans Strains}

All C. elegans strains were maintained on NGM plates at $20^{\circ} \mathrm{C}$ using standard methods unless otherwise statement
(Brenner, 1974). The plasmid SNB-1::pHluorin (SpH, gift from Joshua Kaplan) was injected into wild-type N2 worms and integrated into chromosome by UV/TMP method and backcrossed 6 times with $\mathrm{N} 2$ worms to remove background mutations. The integrated strain was crossed with a RNAi hypersensitive strain KP3948 eri-1(mg366); lin-15b(n744) (Sieburth et al., 2005) to make the transgenic strain eri1(mg366);lin-15b(n744);kanIs8[snb-1::pHluorin] for RNAi screening.

The following strains were used in this study:

bugz-1(tm578), Y25C1A.7(tm2889), T23F11.1(tm3480), F59E12.11(tm3828), $\quad$ C18B12.2(tm1690), $\quad$ RB1445 Y71G12B.11(ok1648), RB861 F41D9.3(ok695), RB1777 F45E4.3(ok2285), VC194 Y76A2A.2(gk107), CB189 unc32(e189), VH624 rhIs13[Punc-119::GFP + dpy-20(+)]; nre-1(hd20); lin-15b(hd126); zxIs6[Punc-17p::chop2(H134R)::yfp, lin-15(+)] (a gift from Dr. Mei Zhen). Single copy worm strain xt11186 kanIs54[bugz-1p::BUGZ-1::gfp, unc-119(+)] II; unc-119(ed9) III

kanEx378[bugz-1p::BUGZ-1::gfp+ rab-3p::mCherry]

kanEx379[bugz-1p::gfp+ rab-3p::mCherry]

bugz-1(tm578); kanEx380[lin-44p::gfp+ rab-3p:: BUGZ-1:: $m$ Cherry]

bugz-1(tm578); kanEx392[lin-44p::gfp+ myo-3p:: BUGZ-1:: mCherry] 


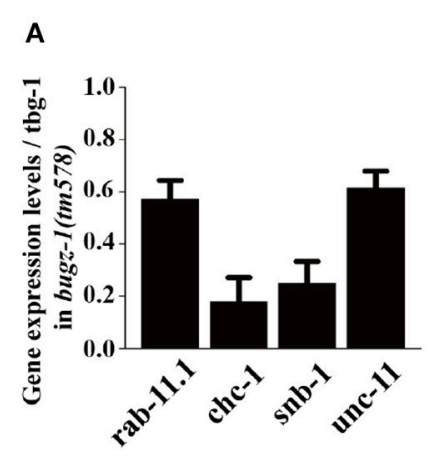

D
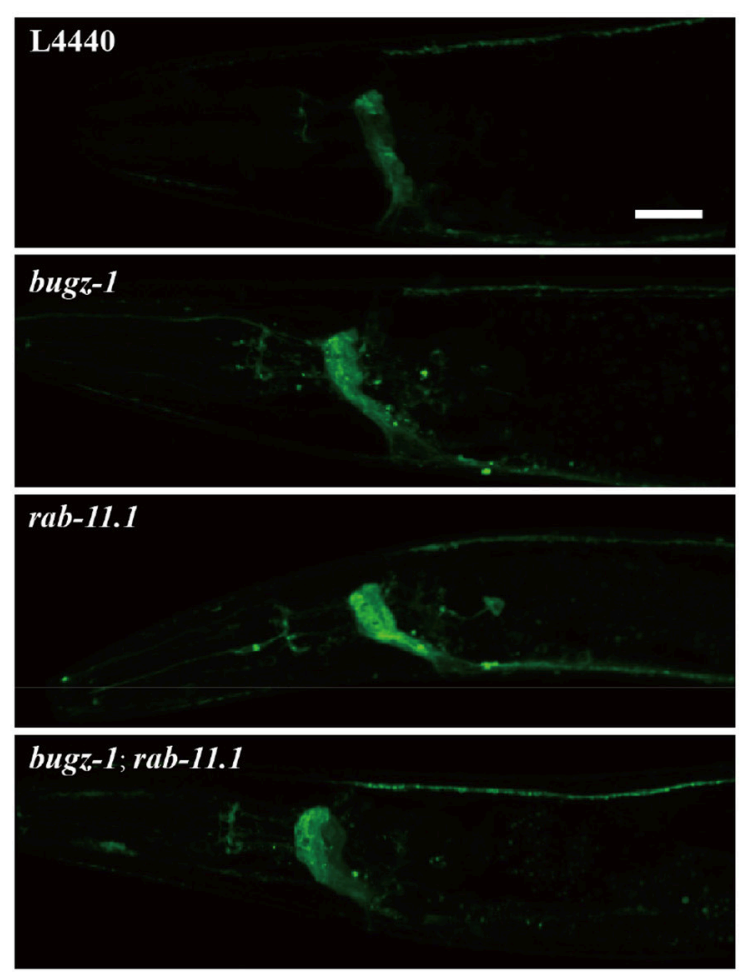
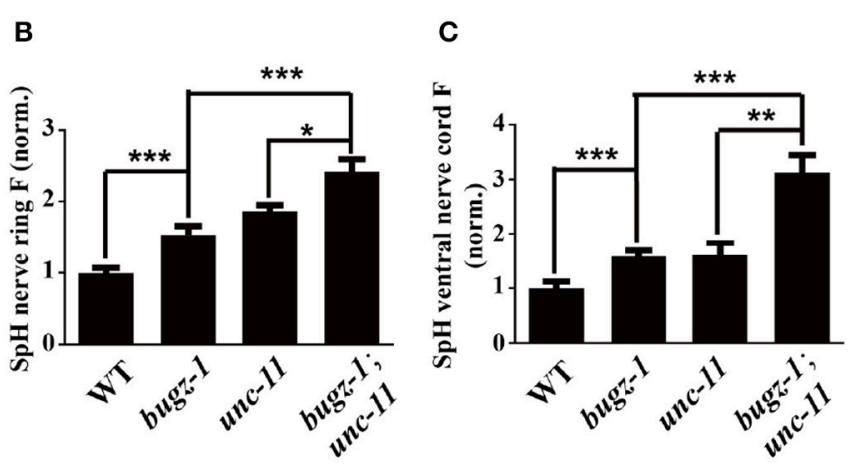

E
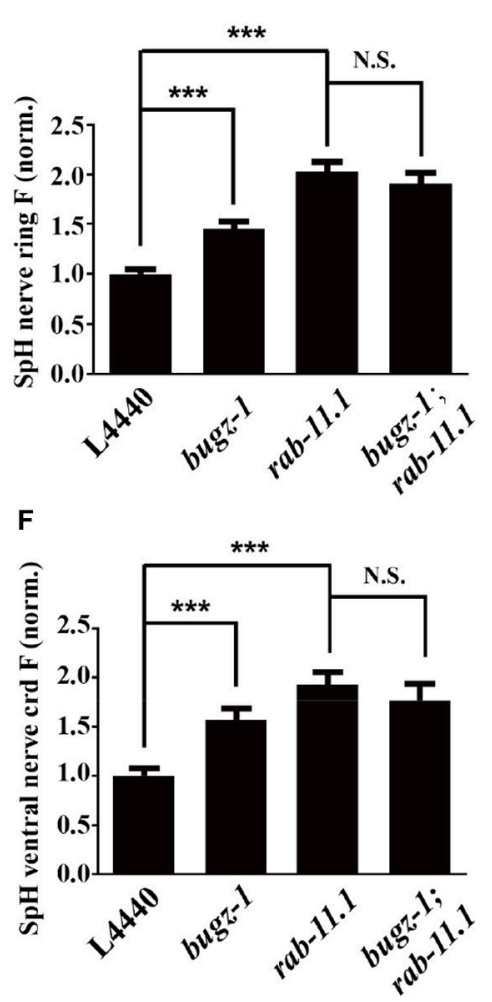

FIGURE 7 | rab-11.1 is a likely direct target of BuGZ-1. (A) qRT-PCR verification of down-regulation of rab-11.1, chc-1, snb-1, and unc-11 transcript levels in bugz-1(tm578) mutant relative to wild type. (B,C) Quantification of SpH fluorescence in the nerve ring (B) and ventral nerve cord (C) of N2, bugz-1, unc-11, and bugz-1;unc-11 worms. $n \geq 14$. (D) Representative confocal images of eri-1(mg366);lin-15b(n744);/s[snb-1::pHluorin] worm treated with L4440, bugz-1, rab-11.1, and bugz-1;rab-11.1 RNAi bacteria. Scale bar indicates $10 \mu \mathrm{m}$. (E,F) Quantification of fluorescence in the nerve ring (E) and ventral nerve cord (F) of indicated RNAi treatment which normalized to the L4440 control. $n \geq 14$. Values that significantly differ from controls are indicated ${ }^{\star} p<0.05 ;{ }^{* \star} p<0.01$; ${ }^{* \star *} p<$ 0.001 by two-tails Student's $t$-test). N.S. indicates no significant difference between genotypes compared. Error bars represent SEM.

bugz-1(tm578);kanEx380[lin-44p::gfp+ rab-3p:: BUGZ-1:: $m$ Cherry]; zxIs6[unc-17p::chop-2(H134R)::yfp, lin-15(+)] bugz-1(tm578);kanEx392[lin-44p::gfp+ myo-3p:: BUGZ-1:: mCherry]; zxIs6[unc-17p::chop-2(H134R)::yfp, lin-15(+)].

\section{RNAi Screen}

Bacterial glycerol stocks of RNAi library was replicated onto LB-agar square plates containing $25 \mu \mathrm{g} / \mathrm{ml}$ carbenicillin and 15 $\mu \mathrm{g} / \mathrm{ml}$ tetracycline using a 96-pin replicator. After overnight culture at $37^{\circ} \mathrm{C}$, bacterial clones were replicated from square plates into $1.2 \mathrm{~mL}$ 96-well plates containing $400 \mu \mathrm{l} \mathrm{LB}$ liquid medium with $25 \mu \mathrm{g} / \mathrm{ml}$ carbenicillin in each well. The RNAi bacterial clones in $1.2 \mathrm{~mL}$ 96-well plates were cultured overnight at $37^{\circ} \mathrm{C}$ with shaking. Added IPTG to bacterial cultures to a final $1 \mathrm{mM}$ concentration to induce transcription of doublestranded RNA and incubated at $37^{\circ} \mathrm{C}$ for $1 \mathrm{~h}$ with shaking. Bacterial clones were spun down and resuspended with 200 $\mu \mathrm{l} \mathrm{S}$-Basal buffer containing $50 \mu \mathrm{g} / \mathrm{ml}$ carbenicillin and $1 \mathrm{mM}$ IPTG. Forty microliters concentrated bacterial suspension was added into each well of standard 96-well plates. RNAi bacteria 
from original 96-well RNAi library were rearranged to make each experimental 96-well plate contained two L4440 controls, one $g f p$ RNAi down-regulated control and one unc-11 RNAi up-regulated control.

Adult hermaphrodite worms were bleached using standard method. Synchronized L1s were washed and resuspended in S-Basal containing $1 \mathrm{mM}$ IPTG, $50 \mu \mathrm{g} / \mathrm{ml}$ carbenicillin, and 0.01\% Tween-20. 5-8 L1s/10 $\mu$ l S-Basal for F1 generation screen or $120 \mathrm{~L} 1 \mathrm{~s} / 10 \mu \mathrm{l}$ for P0 generation screen. Ten microliters worm solution was added into each well of 96-well plates which pre-added $40 \mu \mathrm{l}$ concentrated RNAi bacterial suspension. Experimental 96-well plates containing bacterial clones and L1s were incubated in humid chambers at $20^{\circ} \mathrm{C}$. To increase RNAi efficiency, we used two-generation RNAi treatment for wholegenome screen: synchronized L1 worms were cultured for 6 days, and fluorescent intensity of their progeny were detected by COPAS (Han et al., 2013). The RNAi clones which knocking down reduced the progeny number to less than $20 \%$ of average progeny number of the wells in the same plate were defined as sterile or lethal. We rearranged them into 25 new 96-well plates with controls. For those clones, worms were cultured for 2.5 days and L4-young adult stage of the same generation were detected by COPAS.

\section{Statistical Analysis the Screen Data of COPAS}

We use the relative fluorescent signal (RFS) to represent synaptopHluorin $(\mathrm{SpH})$ signal of each worm. The green fluorescent signals (represent all the $\mathrm{SpH}$ fluorescent signals of the worm) divided by the EXT signals (extinction integral value, represented the worm size) would result in the normalized $\mathrm{SpH}$ signals. $\log _{2}$ transformation was used to transform the fluorescent signals linearly. The median of RFS in each well was used to represent the signal of each well.

$$
\mathrm{RFS}=\log _{2} \frac{\text { Green } \text { fluorescent signals }}{\text { EXT signals }}
$$

Robust Z-score (rZ) was used to normalize screen data in all the plates in the whole-genome screen. Robust Z-score was similar to Z-score except that the sample median and sample median absolute deviation (MAD) were used instead of sample mean and sample standard deviation, and thus was not sensitive to outliers (Birmingham et al., 2009).

$$
\mathrm{rZ}=\frac{X-\text { median }(X)}{M A D(X)}
$$

For variable $X, M A D$ is defined as the median of the absolute deviations from the median of $X$.

After robust $\mathrm{Z}$-score normalization, the $\mathrm{rZ}$ score of two repeats were averaged to represent the $\mathrm{SpH}$ fluorescent intensity of the corresponding genes upon RNAi treatment.

In the secondary and cytoplasmic GFP screen, student's $t$ test was used to confirm the positive hits. For each plate, after removing the low repeatability genes (fold change between the two repeats was $>2$ ), one tailed $t$-test was used to identify the experimental RNAi bacteria that were significantly different from L4440 empty control.

Those RNAi bacteria with $p<0.05$ in secondary screen and $>0.05$ in the cytoplasmic GFP screen were considered as positive hits.

\section{Aldicarb-Sensitivity Assay}

Aldicarb-sensitivity experiments were performed on NGM plates containing $1 \mathrm{mM}$ aldicarb as previously described (Wiese et al., 2012). Prepare aldicarb plates $24 \mathrm{~h}$ before the assay and put them at room temperature to let the plates dry. Pick 25-35 L4 worms to a fresh seeded NGM plate $16 \mathrm{~h}$ before the assay. Place a small spot of OP50 in the middle of aldicarb plates and let it dry thoroughly. Young adult animals were transferred to aldicarb plates and tested for paralysis every $10 \mathrm{~min}$ for $2 \mathrm{~h}$ with a harsh touch on the head. Worms failed to response to the touch were identified as paralyzed.

\section{Constructs and Transgenes}

All expression plasmids were based on the pPD95.75 vector unless otherwise statement. The putative bugz-1 promoter region including $5 \mathrm{~kb}$ upstream of the gene bugz-1. bugz-1p::gfp fusion construct was generated by PCR to amplify the bugz-1 promoter region. The PCR product of bugz-1 promoter was digested with $\mathrm{XmaI}$ and AgeI-HF restriction enzymes and ligated into the pPD95.75 vector. Based on the highly consistency of functional domains between the two isoforms of bugz-1, we cloned the long isoform of bugz-1 for all the rescue studies. BuGZ-1 cDNA was cloned from a cDNA library using primers targeted to the start and stop codons of the long isoform, bugz-1b. To generate bugz-1p:: gfp, we cloned bugz-1 cDNA into bugzp$1:: G F P$ using AgeI single restriction enzyme. A $1.3 \mathrm{~kb}$ rab-3 promoter was used to drive bugz-1 expression in nervous system and a $1.2 \mathrm{~kb}$ myo-3 promoter was used to drive bugz-1 expression in body muscles. bugz-1 cDNA was digested by AgeI single restriction enzyme and then inserted into rab-3p::mCherry or myo-3p::mCherry to generate rab-3p::BUGZ-1::mCherry or myo$3 p:: B U G Z-1:: m$ Cherry. GFP or mCherry was fused to the Cterminus of bugz-1 cDNA as a reporter. Transgenic worm strains were obtained by microinjection of corresponding plasmids with Plin-44::gfp or Prab-3::mCherry as a marker. For expression analysis, constructs were injected at $80 \mathrm{ng} / \mu \mathrm{l}$. For rescue assays, constructs were injected at $10 \mathrm{ng} / \mu \mathrm{l}$. All the markers used were injected at $30 \sim 50 \mathrm{ng} / \mu \mathrm{l}$.

\section{Single Copy Insertion}

Transgenic worms were generated by injection constructs into EG4322 [ttTi5605; unc-119(ed9)] animals (Frøkjaer-Jensen et al., 2008). The standard injection mix consisted of $50 \mathrm{ng} / \mu \mathrm{l} \mathrm{bugz-}$ $1:: g f p$, unc-119(+) repair template, $50 \mathrm{ng} / \mu \mathrm{l}$ Mos1 transposase pJL43.1(Pglh-2::transposase), $5 \mathrm{ng} / \mu \mathrm{l}$ myo-3p::mCherry which as a negative marker. Injected animals were transferred to NGM plates, one worm per plate. Individual injected worms were allowed to exhaust the food source of each plate. Once starved, L1 progeny were screened for insertion events with GFP 
fluorescence and wild-type movement but lack of co-injection marker expression.

\section{Image Analysis}

All the images were obtained using a FV 1000 laser scanning confocal microscope (Olympus). Confocal images were captured using a 60X objective with NA 1.4 at $1 \mathrm{x}$ or $2 \mathrm{x}$ digital zoom. Worms were immobilized with $30 \mathrm{mM} \mathrm{NaN}_{3}$ (Sigma) on agarose pads. For quantitative analysis, images were acquired in young adult worms and maximum fluorescent intensity of Z-series was stacked. Background fluorescence was subtracted before analysis. $P$-values were calculated by student $t$-tests. Images were quantified and analyzed using FV10-ASW Viewer and Image $\mathrm{J}$. For fluorescent analysis of $\mathrm{SpH}$ signal in the nerve ring and ventral nerve cord, average of stacked maximum fluorescent intensity were calculated to represent florescent intensity. For fluorescent analysis in the dorsal nerve cord, florescent intensity of puncta, and inter-puncta were measured using Igor Pro software.

\section{Locomotion Analyses}

Locomotion experiments were performed by capturing movies of animals free moving on NGM plates with fresh seeded $E$. Coli OP50 8-12 h before. Fifteen young adult animals (12-14 $\mathrm{h}$ after L4 stage) were transferred to each $60 \mathrm{~mm}$ NGM plate. Ten min after transfer, a 1 min movie of animal moving was recorded using a digital camera installed on a Zeiss dissecting microscope. Movies of locomotion behaviors were analyzed by the "Imaging the Behavior of Nematodes (iBeN)" system developed in our lab. Software of $\mathrm{iBeN}$ system is developed based on the computer vision library, OpenCV (Open Source Computer Vision). Locomotion rates and movement status were achieved automatically by iBeN system.

\section{Electrophysiology}

Electrophysiology assays were performed at the neuromuscular junctions of dissected C. elegans as previously described (Richmond and Jorgensen, 1999; Kang et al., 2010; Yang et al., 2015). Day 2 adult worms were glued on the surface of Sylgardcoated coverslips using cyanoacrylate-based glue (Zou et al., 2017), and a dorsolateral incision was made using a sharp glass pipette to expose the body wall muscles for recording. Wholecell recordings of ventral body wall muscles were carried out by a HEKA EPC10 amplifier using the Patchmaster software. Recording pipettes were pulled from borosilicate glass capillaries (Sutter Instruments) to a resistance of 3-4 M $\Omega$ on a P-97 micropipette puller (Sutter Instruments). The bath solution contained $145 \mathrm{mM} \mathrm{NaCl}, 2.5 \mathrm{mM} \mathrm{KCl}, 5 \mathrm{mM} \mathrm{CaCl} 2,1 \mathrm{mM}$ $\mathrm{MgCl} 2,20 \mathrm{mM}$ glucose and $10 \mathrm{mM}$ HEPES (325-335 mOsm, $\mathrm{pH}$ 7.3). The pipette solution contained $145 \mathrm{mM} \mathrm{KCl}, 2.5 \mathrm{mM}$ $\mathrm{KCl}, 5 \mathrm{mM} \mathrm{MgCl} 2,0.25 \mathrm{mM} \mathrm{CaCl} 2,10 \mathrm{mM}$ HEPES, $10 \mathrm{mM}$ glucose, and $5 \mathrm{mM}$ EGTA, $5 \mathrm{mM}$ ATP, $0.5 \mathrm{mM}$ GTP (325 335 $\mathrm{mOsm}, \mathrm{pH}$ 7.2). Membrane potential was clamped at $-60 \mathrm{mV}$. For acetylcholine-activated experiments, $500 \mu \mathrm{M}$ acetylcholine was perfused to the bath solution. The zxIs6 strain, in which light-gated cation channel channelrhodopsin-2 (ChR2)-YFP was expressed in cholinergic neurons, was used for recording evoked
EPSCs (Liewald et al., 2008; Yang et al., 2015). All-trans retinal was added to the NGM plates at a final concentration of 2.5 $\mu \mathrm{M}$ to mediate light stimulation of ChR2. L4 animals were transferred to NGM plates containing all-trans retinal and the next generation of 2-day-old hermaphrodite adults were used for electrophysiological studies. Blue light stimulation were performed by LAMBDA XL (Sutter Instruments) with a GFP filter controlled by the Patchmaster software.

\section{RNA Isolation and qPCR Analysis}

RNA was extracted from young adult animals using Trizol reagent (Invitrogen). Total RNA was reversed to cDNA using Reverse Transcription System A3500 (Promega). For quantitative RT-PCR, Bio-Rad CFX96 real-time PCR Amplifier was used to run the cycles. $\mathrm{QPCR}$ was performed in triplicate for three independent biological experiments. Relative gene expression levels were calculated by $\Delta \mathrm{Ct}$ method.

\section{ChIP-Sequencing}

ChIP assays were performed as previously described (Kudron et al., 2013; Kasper et al., 2014). Young adult worms were collected after $50 \mathrm{~h}$ post synchronized L1. Worms were crosslinked in $2 \%$ formaldehyde for $30 \mathrm{~min}$ and then quenched with $1 \mathrm{M}$ Tris 7.5. Worm sample was sonicated to obtain 200$800 \mathrm{bp}$ DNA fragment. $4.4 \mathrm{mg}$ cell extract from the sonicated worm sample was immunoprecipitated with $7.5 \mu \mathrm{g}$ of GoatV$\alpha$ GFP antibody (gift from Kevin White). Deep sequencing was performed on the Illumina Hiseq 2500 platform for the immunoprecipitated DNA fragments and genomic DNA input control. Sequencing consortium version of C. elegans WS235 was used to align reads. Significant binding peaks were called with SPP and IDR algorithms (Landt et al., 2012). The closest coding gene to the peak maximum of a binding site was considered as a target of the transcription factor. The ChIP-seq raw data have been uploaded to the ENCODE wedsite for public viewing and downloading (https://www.encodeproject.org/experiments/ ENCSR450GPA/).

\section{Statistical Analysis}

Data analysis was performed using Excel or Igor 5. All data were presented in mean \pm SEM. Unpaired two-tailed $t$-test was used for data comparison and $P<0.05$ were considered to be statistically significant.

\section{AUTHOR CONTRIBUTIONS}

MH, YY, LK, and TX conceived and designed the experiments. MH, WZ, HC, YY, HZ, SL, HKC, GW, and YC performed molecular genetics, optogenetics, behavioral, and electrophysiological experiments. MH, VR, LK, and TX analyzed and interpreted results. MH, YY, and LK wrote the manuscript and modified by all the other authors.

\section{ACKNOWLEDGMENTS}

We thank Nan Qiao and Jing-Dong J. Han for help with COPAS data analysis. We thank the modERN consortium, especially 
Michelle Kudron and Swapna Samanta, for help with ChIPseqencing, LaDeana Hillier for target calling analysis. We thank Shohei Mitani for providing tm deletion worm strains. We thank the CGC for providing worm strains (P40 OD010440). This work was supported by grants from the Major National Scientific Research Projects of the Ministry of Science and Technology of China (2013CB945603, 2015AA020512), the National Foundation of Natural Science of China (31271180, 31471023),

\section{REFERENCES}

Afuwape, O. A., and Kavalali, E. T. (2016). Imaging synaptic vesicle exocytosisendocytosis with $\mathrm{pH}$-sensitive fluorescent proteins. Methods Mol. Biol. 1474, 187-200. doi: 10.1007/978-1-4939-6352-2_11

Birmingham, A., Selfors, L. M., Forster, T., Wrobel, D., Kennedy, C. J., Shanks, E., et al. (2009). Statistical methods for analysis of high-throughput RNA interference screens. Nat. Methods 6, 569-575. doi: 10.1038/nmeth.1351

Brenner, S. (1974). The genetics of Caenorhabditis elegans. Genetics 77, 71-94.

Culetto, E., Baylis, H. A., Richmond, J. E., Jones, A. K., Fleming, J. T., Squire, M. D., et al. (2004). The Caenorhabditis elegans unc-63 gene encodes a levamisolesensitive nicotinic acetylcholine receptor alpha subunit. J. Biol. Chem. 279, 42476-42483. doi: 10.1074/jbc.M404370200

Dittman, J. S., and Kaplan, J. M. (2006). Factors regulating the abundance and localization of synaptobrevin in the plasma membrane. Proc. Natl. Acad. Sci. U.S.A. 103, 11399-11404. doi: 10.1073/pnas.0600784103

Dupuy, D., Bertin, N., Hidalgo, C. A., Venkatesan, K., Tu, D., Lee, D., et al. (2007). Genome-scale analysis of in vivo spatiotemporal promoter activity in Caenorhabditis elegans. Nat. Biotechnol. 25, 663-668. doi: 10.1038/nbt1305

Frøkjaer-Jensen, C., Davis, M. W., Hopkins, C. E., Newman, B. J., Thummel, J. M., Olesen, S. P., et al. (2008). Single-copy insertion of transgenes in Caenorhabditis elegans. Nat. Genet. 40, 1375-1383. doi: 10.1038/ng.248

Han, M., Chang, H., Zhang, P., Chen, T., Zhao, Y., Zhang, Y., et al. (2013). C13C4.5/Spinster, an evolutionarily conserved protein that regulates fertility in C. elegans through a lysosome-mediated lipid metabolism process. Protein Cell 4, 364-372. doi: 10.1007/s13238-013-3015-Z

Jadiya, P., Fatima, S., Baghel, T., Mir, S. S., and Nazir, A. (2016). A systematic RNAi screen of neuroprotective genes identifies novel modulators of alpha-synucleinassociated effects in transgenic Caenorhabditis elegans. Mol. Neurobiol. 53, 6288-6300. doi: 10.1007/s12035-015-9517-3

Jiang, H., He, X., Wang, S., Jia, J., Wan, Y., Wang, Y., et al. (2014). A microtubuleassociated zinc finger protein, BuGZ, regulates mitotic chromosome alignment by ensuring Bub3 stability and kinetochore targeting. Dev. Cell 28, 268-281. doi: 10.1016/j.devcel.2013.12.013

Kamath, R. S., Fraser, A. G., Dong, Y., Poulin, G., Durbin, R., Gotta, M., et al. (2003). Systematic functional analysis of the Caenorhabditis elegans genome using RNAi. Nature 421, 231-237. doi: 10.1038/nature01278

Kang, L., Gao, J., Schafer, W. R., Xie, Z., and Xu, X. Z. (2010). C. elegans TRP family protein TRP-4 is a pore-forming subunit of a native mechanotransduction channel. Neuron 67, 381-391. doi: 10.1016/j.neuron.2010.06.032

Kasper, D. M., Wang, G. L., Gardner, K. E., Johnstone, T. G., and Reinke, V. (2014). The C. elegans SNAPc component SNPC-4 coats piRNA domains and is globally required for piRNA abundance. Dev. Cell 31, 145-158. doi: 10.1016/j.devcel.2014.09.015

Kim, J. K., Gabel, H. W., Kamath, R. S., Tewari, M., Pasquinelli, A., Rual, J. F., et al. (2005). Functional genomic analysis of RNA interference in C. elegans. Science 308, 1164-1167. doi: 10.1126/science.1109267

Kudron, M., Niu, W., Lu, Z., Wang, G. L., Gerstein, M., Snyder, M., et al. (2013). Tissue-specific direct targets of Caenorhabditis elegans Rb/E2F dictate distinct somatic and germline programs. Genome Biol. 14:r15. doi: 10.1186/gb-2013-14-1-r5

Lackner, M. R., Nurrish, S. J., and Kaplan, J. M. (1999). Facilitation of synaptic transmission by EGL-30 Gq $\alpha$ and EGL- 8 PLC $\beta$ : DAG binding to UNC-13 is required to stimulate acetylcholine release. Neuron 24, 335-346. doi: 10.1016/S0896-6273(00)80848-X and Zhejiang Natural Science Funds for Distinguished Young Scholars of China (LR14C090001).

\section{SUPPLEMENTARY MATERIAL}

The Supplementary Material for this article can be found online at: http://journal.frontiersin.org/article/10.3389/fnmol. 2017.00141/full\#supplementary-material

Landt, S. G., Marinov, G. K., Kundaje, A., Kheradpour, P., Pauli, F., Batzoglou, S., et al. (2012). ChIP-seq guidelines and practices of the ENCODE and modENCODE consortia. Genome Res. 22, 1813-1831. doi: $10.1101 /$ gr.136184.111

Lehner, B., Tischler, J., and Fraser, A. G. (2006). RNAi screens in Caenorhabditis elegans in a 96-well liquid format and their application to the systematic identification of genetic interactions. Nat. Protoc. 1, 1617-1620. doi: 10.1038/nprot.2006.245

Liewald, J. F., Brauner, M., Stephens, G. J., Bouhours, M., Schultheis, C., Zhen, M., et al. (2008). Optogenetic analysis of synaptic function. Nat. Methods 5, 895-902. doi: 10.1038/nmeth.1252

Mahoney, T. R., Liu, Q., Itoh, T., Luo, S., Hadwiger, G., Vincent, R., et al. (2006a). Regulation of synaptic transmission by RAB-3 and RAB-27 in Caenorhabditis elegans. Mol. Biol. Cell 17, 2617-2625. doi: 10.1091/mbc.E05-12-1170

Mahoney, T. R., Luo, S., and Nonet, M. L. (2006b). Analysis of synaptic transmission in Caenorhabditis elegans using an aldicarb-sensitivity assay. Nat. Protoc. 1, 1772-1777. doi: 10.1038/nprot.2006.281

Miesenböck, G., De Angelis, D. A., and Rothman, J. E. (1998). Visualizing secretion and synaptic transmission with $\mathrm{pH}$-sensitive green fluorescent proteins. Nature 394, 192-195. doi: 10.1038/28190

Nonet, M. L., Holgado, A. M., Brewer, F., Serpe, C. J., Norbeck, B. A., Holleran, J., et al. (1999). UNC-11, a Caenorhabditis elegans AP180 homologue, regulates the size and protein composition of synaptic vesicles. Mol. Biol. Cell 10, 2343-2360. doi: $10.1091 / \mathrm{mbc} \cdot 10.7 .2343$

Pulak, R. (2006). Techniques for analysis, sorting, and dispensing of C. elegans on the COPAS ${ }^{\mathrm{TM}}$ flow-sorting system. Methods Mol. Biol. 351, 275-286. doi: 10.1385/1-59745-151-7:275

Richmond, J. E., and Broadie, K. S. (2002). The synaptic vesicle cycle: exocytosis and endocytosis in Drosophila and C. elegans. Curr. Opin. Neurobiol. 12, 499-507. doi: 10.1016/S0959-4388(02)00360-4

Richmond, J. E., and Jorgensen, E. M. (1999). One GABA and two acetylcholine receptors function at the C. elegans neuromuscular junction. Nat. Neurosci. 2, 791-797. doi: 10.1038/12160

Rizo, J., and $\mathrm{Xu}$, J. (2015). The synaptic vesicle release machinery. Annu. Rev. Biophys. 44, 339-367. doi: 10.1146/annurev-biophys-060414-034057

Rual, J. F., Ceron, J., Koreth, J., Hao, T., Nicot, A. S., Hirozane-Kishikawa, T., et al. (2004). Toward improving Caenorhabditis elegans phenome mapping with an ORFeome-based RNAi library. Genome Res. 14, 2162-2168. doi: $10.1101 / g r .2505604$

Sankaranarayanan, S., De Angelis, D., Rothman, J. E., and Ryan, T. A. (2000). The use of pHluorins for optical measurements of presynaptic activity. Biophys. J. 79, 2199-2208. doi: 10.1016/S0006-3495(00)76468-X

Sato, M., Sato, K., Liou, W., Pant, S., Harada, A., and Grant, B. D. (2008). Regulation of endocytic recycling by C. elegans Rab35 and its regulator RME-4, a coated-pit protein. EMBO J. 27, 1183-1196. doi: 10.1038/emboj. 2008.54

Schmitz, C., Kinge, P., and Hutter, H. (2007). Axon guidance genes identified in a large-scale RNAi screen using the RNAi-hypersensitive Caenorhabditis elegans strain nre-1(hd20) lin-15b(hd126). Proc. Natl. Acad. Sci. U.S.A. 104, 834-839. doi: $10.1073 /$ pnas.0510527104

Sieburth, D., Ch'ng, Q., Dybbs, M., Tavazoie, M., Kennedy, S., Wang, D., et al. (2005). Systematic analysis of genes required for synapse structure and function. Nature 436, 510-517. doi: 10.1038/nature03809

Sudhof, T. C. (2004). The synaptic vesicle cycle. Annu. Rev. Neurosci. 27, 509-547. doi: 10.1146/annurev.neuro.26.041002.131412 
Südhof, T. C. (2013). Neurotransmitter release: the last millisecond in the life of a synaptic vesicle. Neuron 80, 675-690. doi: 10.1016/j.neuron.2013.10.022

Sudhof, T. C., and Rizo, J. (2011). Synaptic vesicle exocytosis. Cold Spring Harb. Perspect. Biol 3:a005637. doi: 10.1101/cshperspect.a005637

Tadepally, H. D., Burger, G., and Aubry, M. (2008). Evolution of C2H2zinc finger genes and subfamilies in mammals: species-specific duplication and loss of clusters, genes and effector domains. BMC Evol. Biol. 8:176. doi: 10.1186/1471-2148-8-176

Toledo, C. M., Herman, J. A., Olsen, J. B., Ding, Y., Corrin, P., Girard, E. J., et al. (2014). BuGZ is required for Bub3 stability, Bub1 kinetochore function, and chromosome alignment. Dev. Cell 28, 282-294. doi: 10.1016/j.devcel.2013.12.014

Wang, Y., Gracheva, E. O., Richmond, J., Kawano, T., Couto, J. M., Calarco, J. A., et al. (2006). The $\mathrm{C} 2 \mathrm{H} 2$ zinc-finger protein SYD-9 is a putative posttranscriptional regulator for synaptic transmission. Proc. Natl. Acad. Sci. U.S.A. 103, 10450-10455. doi: 10.1073/pnas.0602073103

Wiese, M., Antebi, A., and Zheng, H. (2012). Regulation of neuronal APL-1 expression by cholesterol starvation. PLoS ONE 7:e32038. doi: 10.1371/journal.pone.0032038

Wu, L. G., Hamid, E., Shin, W., and Chiang, H. C. (2014). Exocytosis and endocytosis: modes, functions, and coupling mechanisms. Annu. Rev. Physiol. 76, 301-331. doi: 10.1146/annurev-physiol-021113-170305
Xie, Z., Long, J., Liu, J., Chai, Z., Kang, X., and Wang, C. (2017). Molecular mechanisms for the coupling of endocytosis to exocytosis in neurons. Front. Mol. Neurosci. 10:47. doi: 10.3389/fnmol.2017.00047

Yang, X., Wang, S., Sheng, Y., Zhang, M., Zou, W., Wu, L., et al. (2015). Syntaxin opening by the MUN domain underlies the function of Munc13 in synaptic-vesicle priming. Nat. Struct. Mol. Biol. 22, 547-554. doi: 10.1038/ nsmb.3038

Zou, W., Cheng, H., Li, S., Yue, X., Xue, Y., Chen, S., et al. (2017). Polymodal responses in C. elegans phasmid neurons rely on multiple intracellular and intercellular signaling pathways. Sci. Rep. 7:42295. doi: 10.1038/srep42295

Conflict of Interest Statement: The authors declare that the research was conducted in the absence of any commercial or financial relationships that could be construed as a potential conflict of interest.

Copyright $\odot 2017$ Han, Zou, Chang, Yu, Zhang, Li, Cheng, Wei, Chen, Reinke, Xu and Kang. This is an open-access article distributed under the terms of the Creative Commons Attribution License (CC BY). The use, distribution or reproduction in other forums is permitted, provided the original author(s) or licensor are credited and that the original publication in this journal is cited, in accordance with accepted academic practice. No use, distribution or reproduction is permitted which does not comply with these terms. 